\title{
Chaos analyses in both phases of QED and QCD
}

\author{
Tamás S. Biróa ${ }^{\text {, Natascha Hörmann }}{ }^{\mathrm{b}}$, Harald Markum ${ }^{\mathrm{b}}$, and Rainer Pullirsch ${ }^{\mathrm{b}}$ \\ ${ }^{a}$ Research Institute for Particle and Nuclear Physics, Pf.49, H-1525 Budapest, Hungary \\ ${ }^{\mathrm{b}}$ Institute for Nuclear Physics, TU-Wien, Wiedner Hauptstraße 8-10, A-1040 Vienna, Austria
}

We analyze the leading Lyapunov exponents of $\mathrm{U}(1)$ and $\mathrm{SU}(2)$ gauge field configurations on the lattice which are initialized by quantum Monte Carlo simulations. We find that configurations in the strong coupling phase are substantially more chaotic than in deconfinement.

\section{Motivation}

The study of chaotic dynamics of classical field configurations in field theory finds its motivation in phenomenological applications as well as for the understanding of basic principles. The role of chaotic field dynamics for the confinement of quarks is a longstanding question. Here, we analyze the leading Lyapunov exponents of compact $\mathrm{U}(1)$ and of $\mathrm{SU}(2)$-Yang-Mills field configurations on the lattice. The real-time evolution of the classical field equations was initialized from Euclidean equilibrium configurations created by quantum Monte Carlo simulations. This way we expect to see a coincidence between the strong coupling phase and the strength of chaotic behavior in lattice simulations.

After reviewing essential definitions of the physical quantities describing chaos and their computation in lattice gauge theory [1] we outline our method for the extraction of starting configurations of a three dimensional Hamiltonian dynamics from four dimensional Euclidean field configurations. Our results are then presented by showing an example of the exponential divergence of small initial distances between nearby field configurations. It is followed by a detailed study of the maximal Lyapunov exponent and average plaquette energy as a function of the coupling strength.

\section{Classical chaotic dynamics}

Chaotic dynamics in general is characterized by the spectrum of Lyapunov exponents. These exponents, if they are positive, reflect an exponential divergence of initially adjacent configurations. In case of symmetries inherent in the Hamiltonian of the system there are corresponding zero values of these exponents. Finally negative exponents belong to irrelevant directions in the phase space: perturbation components in these directions die out exponentially. Pure gauge fields on the lattice show a characteristic Lyapunov spectrum consisting of one third of each kind of exponents [1]. This fact reflects the elimination of longitudinal degrees of freedom of the gauge bosons. Assuming this general structure of the Lyapunov spectrum we investigate presently its magnitude only, namely the maximal value of the Lyapunov exponent, $L_{\max }$.

The general definition of the Lyapunov exponent is based on a distance measure $d(t)$ in phase space,

$L:=\lim _{t \rightarrow \infty} \lim _{d(0) \rightarrow 0} \frac{1}{t} \ln \frac{d(t)}{d(0)}$.

In case of conservative dynamics the sum of all Lyapunov exponents is zero according to Liouville's theorem,

$\sum L_{i}=0$.

We utilize the gauge invariant distance measure consisting of the local differences of energy densities between two field configurations on the lattice:

$d:=\frac{1}{N_{P}} \sum_{P}\left|\operatorname{tr} U_{P}-\operatorname{tr} U_{P}^{\prime}\right|$. 
Here the symbol $\sum_{P}$ stands for the sum over all $N_{P}$ plaquettes, so this distance is bound in the interval $(0,2 N)$ for the group $\mathrm{SU}(\mathrm{N}) . U_{P}$ and $U_{P}^{\prime}$ are the familiar plaquette variables, constructed from the basic link variables $U_{x, i}$,

$U_{x, i}=\exp \left(a A_{x, i}^{c} T^{c}\right)$,

located on lattice links pointing from the position $x=\left(x_{1}, x_{2}, x_{3}\right)$ to $x+a e_{i}$. The generators of the group are $T^{c}=-i g \tau^{c} / 2$ with $\tau^{c}$ being the Pauli matrices in case of $\mathrm{SU}(2)$ and $A_{x, i}^{c}$ is the vector potential. The elementary plaquette variable is constructed for a plaquette with a corner at $x$ and lying in the $i j$-plane as

$U_{x, i j}=U_{x, i} U_{x+i, j} U_{x+j, i}^{\dagger} U_{x, j}^{\dagger}$.

It is related to the magnetic field strength $B_{x, k}^{c}$ :

$U_{x, i j}=\exp \left(\varepsilon_{i j k} a B_{x, k}^{c} T^{c}\right)$.

The electric field strength $E_{x, i}^{c}$ is related to the canonically conjugate momentum $P_{x, i}=\dot{U}_{x, i}$ via

$E_{x, i}^{c}=\frac{2 a}{g^{3}} \operatorname{tr}\left(T^{c} \dot{U}_{x, i} U_{x, i}^{\dagger}\right)$.

\section{Initial states prepared by quantum Monte Carlo}

The Hamiltonian of the lattice gauge field system can be casted into the form

$H=\sum\left[\frac{1}{2}\langle P, P\rangle+1-\frac{1}{4}\langle U, V\rangle\right]$.

Here the scalar product between group elements stands for $\langle A, B\rangle=\frac{1}{2} \operatorname{tr}\left(A B^{\dagger}\right)$. The staple variable $V$ is a sum of triple products of elementary link variables closing a plaquette with the chosen link $U$. This way the Hamiltonian is formally written as a sum over link contributions and $V$ plays the role of the classical force acting on the link variable $U$. The naive equations of motion following from this Hamiltonian, however, have to be completed in order to fulfill the constraints $\langle U, U\rangle=1$

$\langle P, U\rangle=0$.

The following finite time step recursion formula:

$U^{\prime}=U+d t\left(P^{\prime}-\varepsilon U\right)$,

$P^{\prime}=P+d t\left(V-\mu U+\varepsilon P^{\prime}\right)$, with the Lagrange multipliers

$\varepsilon=\left\langle U, P^{\prime}\right\rangle$,

$\mu=\langle U, V\rangle+\left\langle P^{\prime}, P^{\prime}\right\rangle$

conserves the Noether charge belonging to the Gauss law,

$\Gamma=\sum_{+} P U^{\dagger}-\sum_{-} U^{\dagger} P$.

Here the sums indicated by + run over links starting from, and those by - ending at a given site $x$, where the Noether charge $\Gamma$ is defined. The above algorithm is written in an implicit form, but it can be casted into explicit steps, so no iteration is necessary [2].

Initial conditions chosen randomly with a given average magnetic energy per plaquette have been investigated in past years. In the $\mathrm{SU}(2)$ case, a linear scaling of the maximal Lyapunov exponent with the total energy of the system has been established for different lattice sizes and coupling strengths [1]. In the present study we prepare the initial field configurations from a standard four dimensional Euclidean Monte Carlo program on a $12^{3} \times 4$ lattice varying the inverse gauge coupling $\beta$ [3].

We relate such four dimensional Euclidean lattice field configurations to Minkowskian momenta and fields for the three dimensional Hamiltonian simulation by the following approach:

First we fix a time slice of the four dimensional lattice. We denote the link variables in the three dimensional sub-lattice by $U^{\prime}=U_{i}(x, t)$. Then we build triple products on attached handles in the positive time direction,

$U^{\prime \prime}=U_{4}(x, t) U_{i}(x, t+a) U_{4}^{\dagger}(x+a, t)$. We obtain the canonical variables of the Hamiltonian system by using

$P=\left(U^{\prime \prime}-U^{\prime}\right) / d t$

$U \propto\left(U^{\prime \prime}+U^{\prime}\right)$.

Finally $U$ is normalized to $\langle U, U\rangle=1$.

This definition constructs the momenta according to a simple definition of the timelike covariant derivative. The multiplication with the link variables in time direction can also be viewed as a gauge transformation to $U_{4}(x, t)=1$, i.e. $A_{0}=0$ Hamiltonian gauge. 


\section{Chaos and confinement}

We start the presentation of our results with a characteristic example of the time evolution of the distance between initially adjacent configurations. An initial state prepared by a standard four dimensional Monte Carlo simulation is evolved according to the classical Hamiltonian dynamics in real time. Afterwards this initial state is rotated locally by group elements which are chosen randomly near to the unity. The time evolution of this slightly rotated configuration is then pursued and finally the distance between these two evolutions is calculated at the corresponding times. A typical exponential rise of this distance followed by a saturation can be inspected in Fig. 1 from an example of $\mathrm{U}(1)$ gauge theory in the confinement phase and in the Coulomb phase. While the saturation is an artifact of the compact distance measure of the lattice, the exponential rise (the linear rise of the logarithm) can be used for the determination of the leading Lyapunov exponent. The naive determination and more sophisticated rescaling methods lead to the same result.

The main result of the present study is the dependence of the leading Lyapunov exponent $L_{\max }$ on the inverse coupling strength $\beta$, displayed in Fig. 2 for a statistics of 100 independent U(1) configurations. As expected the strong coupling phase, where confinement of static sources has been established many years ago by proving the area law behavior for large Wilson loops, is more chaotic. The transition reflects the critical coupling to the Coulomb phase. Furthermore the maximal Lyapunov exponent scatters more pronounced than the average energy per plaquette. Fig. 3 shows the somewhat smoother transition of the energy per plaquette as a function of the inverse coupling strength. Fig. 1 depicts the correlation of the Lyapunov exponents and the plaquette energies for $100 \mathrm{U}(1)$ configurations. The blank area is indicative of the transition point being presumable of first order.

We now turn to a comparison of expectation values of $\mathrm{U}(1)$ and $\mathrm{SU}(2)$ theory. Fig. $\mathrm{f}$ exhibits the averaged leading Lyapunov exponent between the strong and the weak coupling regime. The smoother fall-off of the SU(2) Lyapunov expo-
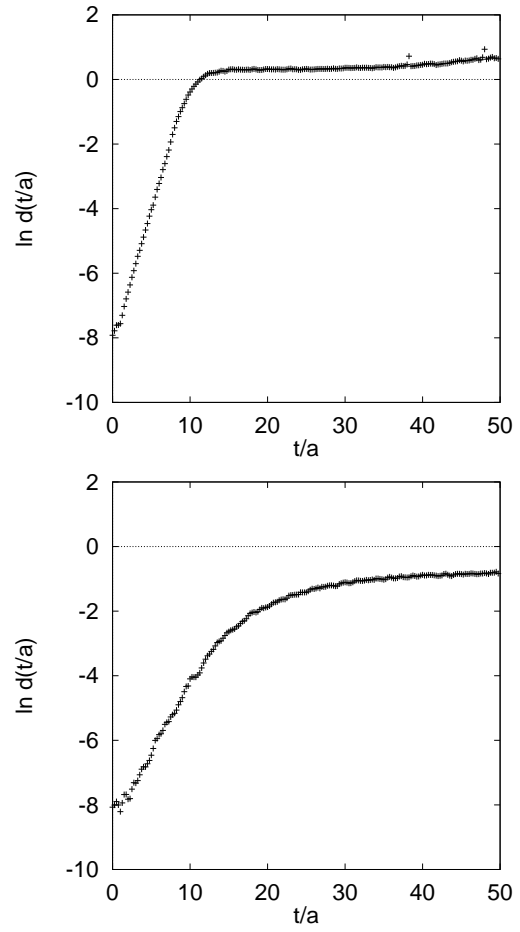

Figure 1. Exponentially diverging distance of initially adjacent $\mathrm{U}(1)$ field configurations on a $12^{3}$ lattice prepared at $\beta=0.9$ in the confinement phase (top) and at $\beta=1.1$ in the Coulomb phase (bottom).

nent reflects the second order of the finite temperature transition to a Debye screened phase of free quarks. Fig. 6 compares the averaged plaquette energies of both gauge theories signaling the different order of their phase transitions.

Fig. 7 shows the energy dependence of the Lyapunov exponents for both theories. One observes an approximately linear relation for the $\mathrm{SU}(2)$ case while a quadratic relation is suggested for the $\mathrm{U}(1)$ theory in the weak coupling regime. From scaling arguments one expects a functional relationship between the Lyapunov exponent and the energy [1, 4]

$L(a) \propto a^{k-1} E^{k}(a)$,

with the exponent $k$ being crucial for the continuum limit of the classical field theory. A value of $k<1$ leads to a divergent Lyapunov exponent, while $k>1$ yields a vanishing $L$ in the contin- 


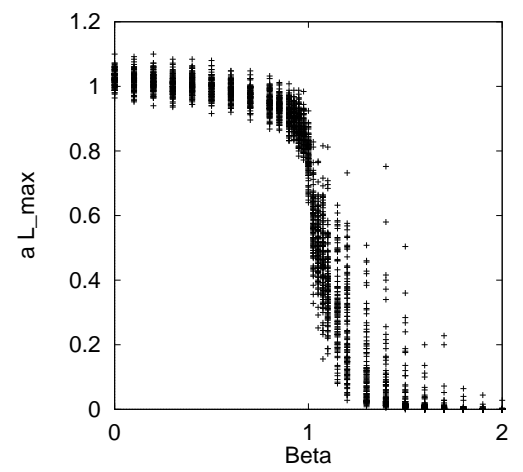

Figure 2. Transition of the leading Lyapunov exponents from $100 \mathrm{U}(1)$ configurations as a function of the inverse coupling strength $\beta$.

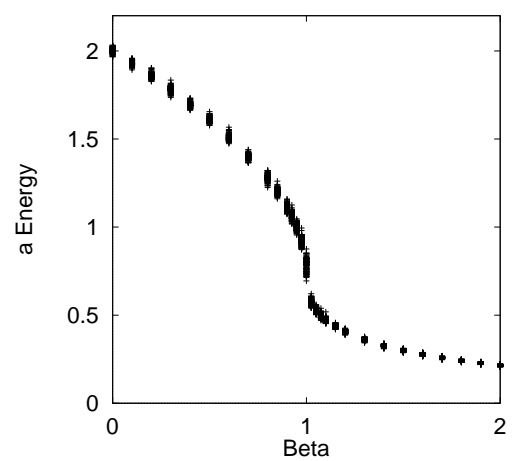

Figure 3. Transition of the plaquette energy as in Fig. 2.

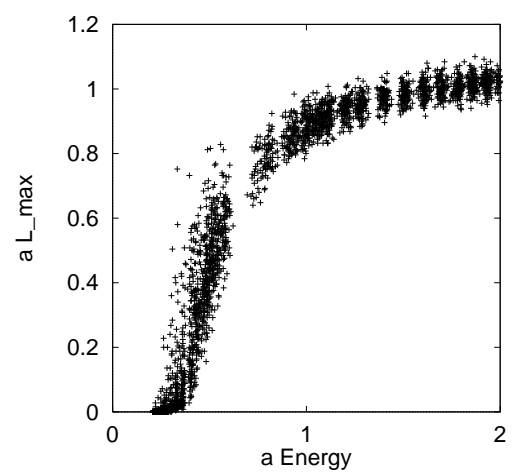

Figure 4. Scatter plots of Lyapunov exponents and plaquette energies for $100 \mathrm{U}(1)$ configurations. uum. The case $k=1$ is special leading to a finite non-zero Lyapunov exponent. Our analysis of the scaling relation (14) gives evidence, that the classical compact $\mathrm{U}(1)$ lattice gauge theory has $k \approx 2$ and with $L(a) \rightarrow 0$ a regular continuum theory. The non-Abelian $\mathrm{SU}(2)$ lattice gauge theory signals $k \approx 1$ and stays chaotic approaching the continuum.

Summarizing we investigated the classical chaotic dynamics of $\mathrm{U}(1)$ and $\mathrm{SU}(2)$ lattice gauge field configurations prepared by quantum Monte Carlo simulation. The maximal Lyapunov exponent shows a pronounced transition as a function of the coupling strength. Both for QED and QCD we find that configurations in the strong coupling phase are substantially more chaotic than in the weak coupling regime. Our results demonstrate that chaos is present when particles are confined, but it persists partly also into the Coulomb and quark-gluon-plasma phase. So far the situation for the gauge fields. An independent analysis of the fermion fields yields compatible results with respect to quantum chaos [5].

\section{Acknowledgments}

This work has been supported by the Hungarian National Scientific Fund under the project OTKA T019700 as well as by the Joint American Hungarian Scientific Fund TeT MAKA 649.

\section{REFERENCES}

1. T.S. Biró, S.G. Matinyan and B. Müller: Chaos and Gauge Field Theory, World Scientific, Singapore, 1995.

2. T.S. Biró, Int. J. Mod. Phys. C6 (1995) 327.

3. T.S. Biró, M. Feurstein and H. Markum, APH Heavy Ion Physics 7 (1998) 235.

4. L. Casetti, R. Gatto and M. Pettini, J. Phys. A32 (1999) 3055; H.B. Nielsen, H.H. Rugh and S.E. Rugh, ICHEP96 1603, hepth/9611128; B. Müller, chao-dyn/9607001; H.B. Nielsen, H.H. Rugh and S.E. Rugh, chao-dyn/9605013.

5. R. Pullirsch, K. Rabitsch, T. Wettig and H. Markum, Phys. Lett. B427 (1998) 119; B.A. Berg, H. Markum and R. Pullirsch, Phys. Rev. D59 (1999) 097504. 

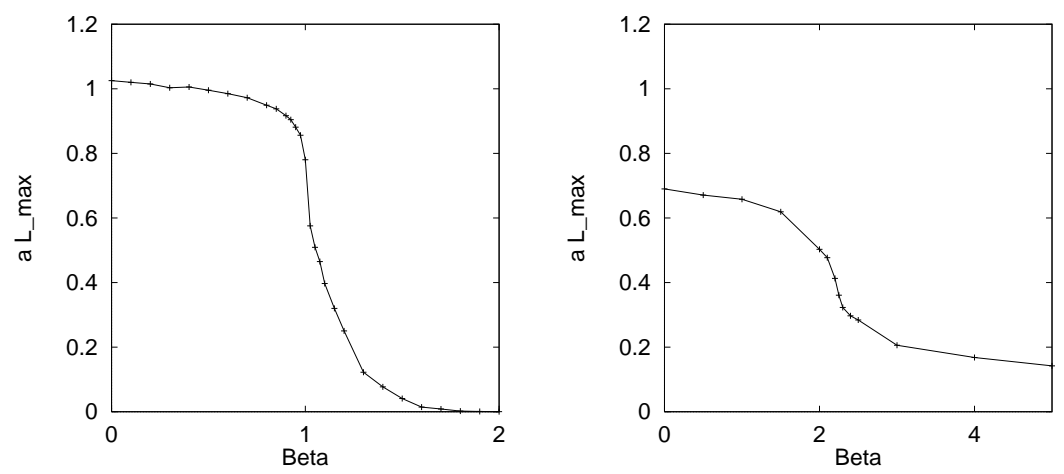

Figure 5. Comparison of the average maximal Lyapunov exponent in $\mathrm{U}(1)$ gauge theory with $\beta=1 / g^{2}$ (left) and in $\mathrm{SU}(2)$ gauge theory with $\beta=4 / g^{2}$ (right) when crossing from the strong to the weak coupling phase.
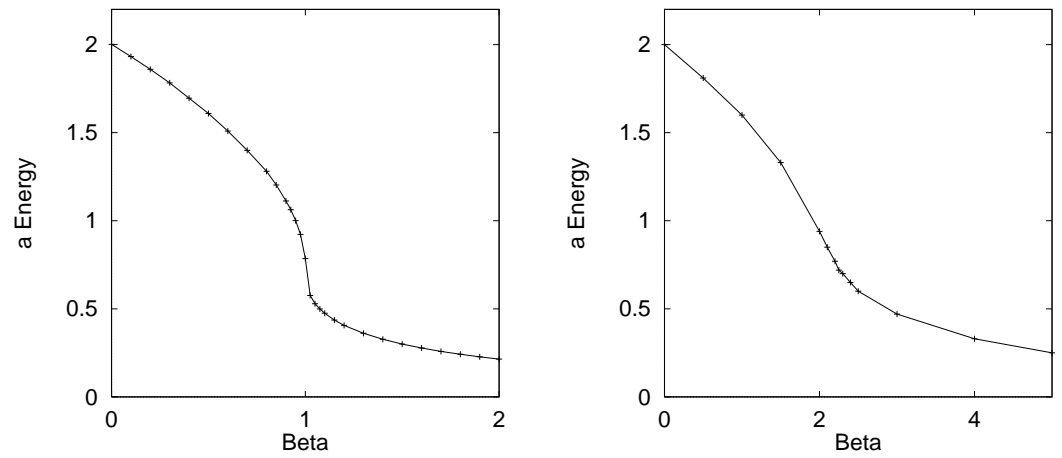

Figure 6. Comparison of the average plaquette energy as in Fig. 5.
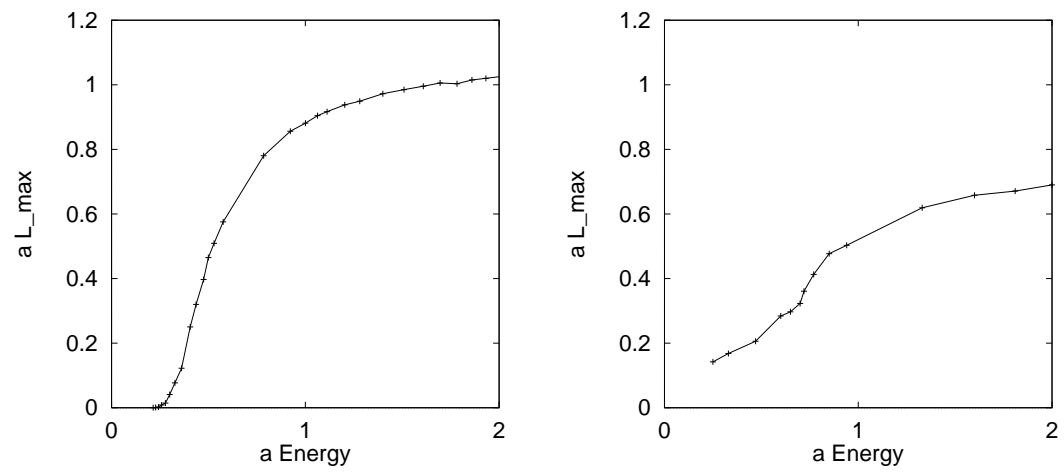

Figure 7. Comparison of average maximal Lyapunov exponents as a function of the scaled average energy per plaquette $a g^{2} E$. The U(1) theory (left) shows an approximately quadratic behavior in the weak coupling regime whereas the $\mathrm{SU}(2)$ theory (right) is approximately linear. 